\title{
The protective effects of dexmedetomidine on liver injury- -induced myocardial ischemia reperfusion
}

\author{
Erer $\mathrm{D}^{1}$, Ozer $\mathrm{A}^{1}$, Arslan $\mathrm{M}^{2}$, Oktar $\mathrm{GL}^{1}$, Iriz $\mathrm{E}^{1}$, Elmas $\mathrm{C}^{3}$, Zor $\mathrm{MH}^{1}$, Tatar $\mathrm{T}^{1}$, Goktas $\mathrm{G}^{3}$ \\ Department of Cardiovascular Surgery, Faculty of Medicine, Gazi University, Ankara, Turkey. \\ marslan36@yahoo.com
}

\begin{abstract}
Purpose: The aim of this study was to evaluate the effect of dexmedetomidine $(100 \mu \mathrm{g} / \mathrm{kg}$-ip) on liver injury-induced myocardial ischemia and reperfusion (IR) in rats.

Materials and methods: Twenty-four Wistar Albino rats were separated into four groups. There were four experimental groups (Group C (Control; $n=6$ ), Group IR (ischemia-reperfusion, $n=6$ ), Group D (Dexmedetomidine; $\mathrm{n}=6$ ) that underwent left thoracotomy and received ip dexmedetomidine without IR administered via $100 \mu \mathrm{g} /$ $\mathrm{kg}$ ip route 30 minutes before ligating the left coronary artery, and Group IR-D (IR-Dexmedetomidine; $n=6$ ). A small plastic snare was threaded through the ligature and placed in contact with the heart. To produce IR, a branch of the left coronary artery was occluded for $30 \mathrm{~min}$ followed by two hours of reperfusion. However, after the above procedure, the coronary artery was not occluded or reperfused in the control rats. At the end of the study, liver tissue was obtained for histochemical and immunohistochemical determination.

Some part of tissue samples were stained with Masson-trichrome for the evaluation of ultrastructural changes and inducible nitric oxide synthase (iNOS) expression was evaluated in other part of samples for immunohistochemical examination.

Results: Histopathological changes were detected in Group IR when compared with Group C. iNOS expression was found to be increased and stronger particularly in the vascular wall, perisinusoidal space and hepatocytes around vena centralis in this group compared to the control group. Perivascular oedema was detected to be decreased in Group IR-D compared to Group IR. It was also observed that the impairment in the radial arrangement of hepatocytes significantly recovered in Group IR-D. The immunoreactivity was found to be significantly decreased in the assessment of iNOS expression in the same group when compared with Group IR. Conclusion: Administration of dexmedetomidine ameliorates liver injury induced by myocardial ischemia and reperfusion (Fig. 8, Ref. 33). Text in PDF www.elis.sk.

Key words: dexmedetomidine, myocardial ischemia reperfusion, remote organ, liver injury, i-NOS, rat.
\end{abstract}

Cardiac surgery with cardiopulmonary bypass inevitably causes a systemic inflammatory response and ischemia-reperfusion (IR) injury affecting multiple organs $(1,2)$. Hepatic injury in cardiac surgery is a rare complication but it is associated with significant morbidity and mortality (3).

One of the important remote organ injuries in myocardial IR may be liver injury, which is believed to be a consequence of free-radical generation in the liver (4-6). The incidence of liver injury is increasing worldwide. Viral infection, alcohol or drug toxicity, and many other factors including IR can cause damage to hepatocytes. Therefore, these factors may cause inflammatory and oxidant reactions in the liver (7-10).

It is possible that IR injury can lead to remote organ injury, including liver damage (11).

${ }^{1}$ Department of Cardiovascular Surgery, Faculty of Medicine, Gazi University, Ankara, Turkey, ${ }^{2}$ Department of Anesthesiology, Faculty of Medicine, Gazi University, Ankara, Turkey, and ${ }^{3}$ Department of Histology and Embryology, Faculty of Medicine, Gazi University, Ankara, Turkey

Address for correspondence: M. Arslan, Dr, Gazi University Medical Faculty, Department of Anesthesiology and Reanimation, 06510 Ankara,Turkey.

Phone: +90.312.2026739, Fax: +90.312.2024166
Dexmedetomidine, a selective and potent $\alpha 2$ - adrenoceptor agonist, was approved by the U.S. Food and Drug Administration in 1999 for sedation of patients hospitalized in intensive care settings. Since then, a growing number of research articles have emerged reporting other possible indications such as regional and general anesthesia $(12,13)$. Dexmedetomidine was reported to be effective in protecting against focal ischemia in rabbits, in cardiac and kidney IR injury in rats, and in incomplete forebrain ischemia in rats (1417). Despite its increased clinical use, many times in critically ill patients, the effects of dexmedetomidine on liver injury induced by left coronary artery (LAD) IR have not been yet investigated (18).

The aim of this study was to investigate histochemical and immunohistochemical changes and the preventive role of dexmedetomidine against these changes in remote organ liver of rats during an experimental model of myocardial IR injury.

\section{Materials and methods}

Animals and experimental protocol

The experiments were performed in adherence to National Institutes of Health guidelines on the use of experimental animals. Twenty-four male Wistar rats, weighing from 250 to $350 \mathrm{~g}$, were housed at 
constant temperature under $12 / 12 \mathrm{~h}$ periods of light and dark exposure. Animals were allowed access to standard rat chow and water $a d$ libitum during the acclimatization period of at least $5 \mathrm{~d}$ prior to being used in these experiments. The protocols of this experimental study were approved by the Animal Ethics Committee of Gazi University.

The rats were anesthetized with ketamine ( $80 \mathrm{mg} / \mathrm{kg}$ ip) and xylazine ( $5 \mathrm{mg} / \mathrm{kg}$ i.p.) The trachea was cannulated for artificial respiration. The chest was shaved, and each animal was fixed in a supine position on the operating table. The chest was opened by left thoracotomy followed by sectioning the fourth and fifth ribs about $2 \mathrm{~mm}$ to the left of the sternum. Positive-pressure artificial respiration was started immediately with room air, using a volume of 1.5 $\mathrm{ml} / 100 \mathrm{~g}$ body weight at a rate of 60 strokes/min. Sodium heparin (500 IU $/ \mathrm{kg}$ ) was administered through the tail peripheral vein.

After the pericardium was incised, the heart was exteriorized by gentle pressure on the right side of the rib cage. An 8/0 silk suture attached to a $10-\mathrm{mm}$ micropoint reverse-cutting needle was quickly placed under the left main coronary artery. The heart was then carefully replaced in the chest and the animal was allowed to recover for $20 \mathrm{~min}$.

There were four experimental groups (Group C (Control; $n=6)$, Group IR (ischemia-reperfusion, $\mathrm{n}=6$ ), Group D (Dexmedetomidine; $n=6$ ) that underwent left thoracotomy and received ip Dexmedetomidine without IR (Precedex $100 \mu \mathrm{g}, / 2 \mathrm{ml}$, Abbott ${ }^{\mathbb{R}}$, Abbott Laboratory, North Chicago, Illinois, USA) administrated via $100 \mu \mathrm{g} / \mathrm{kg}$ intraperitoneal route 30 minutes before ligating the $\operatorname{LAD}(19)$, and Group IR-D(IR-Dexmedetomidine; $\mathrm{n}=6$ ). A small plastic snare was threaded through the ligature and placed in contact with the heart. The artery could then be occluded by applying tension to the ligature (30 $\mathrm{min}$ ), and reperfusion was achieved by releasing the tension (120 $\mathrm{min}$ ) (11). However, after the above procedure, the coronary artery was not occluded or reperfused in the control rats. All rats were sacrificed and the liver tissues were quickly removed after $150 \mathrm{~min}$.

\section{Histopathological evaluation}

All tissues were transferred immediately to $10 \%$ formal saline for 72 hours, dehydrated in graded alcohols and embedded

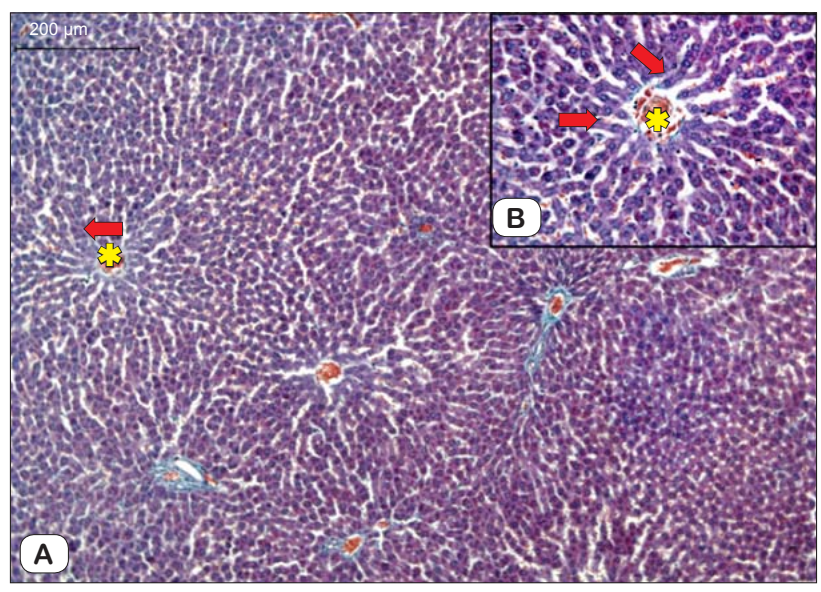

Fig. 1 A,B. Hepatic tissue in the control group *: Vena centralis, $\rightarrow$ : Hepatic chords (Masson-Trichrome AX100,BX400). in paraffin wax. 4- $\mu \mathrm{m}$ sections were cut on a microtome (Leica SM 2000, Germany) and mounted on polylizine-coated sides (Menzel-Glaser, Braunschweig, Germany). Some part of tissue samples were stained with Masson-trichrome for the evaluation of ultrastructural changes, and inducible nitric oxide synthase (iNOS) expression was evaluated in other part of samples for immunohistochemical examination. The slides were incubated overnight at $37^{\circ} \mathrm{C}$. Then for $1 \mathrm{~h}$ at $60{ }^{\circ} \mathrm{C}$, they were de-waxed by two chances of xylene (10 minutes each) and placed in $100 \%, 96 \%$ and $80 \%$ ethanol for 10 minutes each, followed by two 5-minute changes of distilled water. Then tissues were circumscribed with a pap pen (Super PAP Pen, PN IM3580, Becman Coulter Company, France). After washing with distilled water and with phosphate buffer saline (PBS, $\mathrm{pH}=7.4$ ), endogenous enzymes were blocked using hydrogen peroxide for 10 minutes. Following a PBS wash, slides were blocked using ready to use Ultra block (Cat: 859043, Lot: 903532A, İnvitrogen, USA) for 10 minutes, prior to application of a $1: 50$ concentration of i-NOS (Cat: sc-651, Lot: H1308, Santa Cruz, USA) for 1 hour. Two PBS rinses preceded secondary antibody (Cat: 859043, Lot: 903532A, Invitrogen, USA) application for 10 minutes. After washing with PBS, slides were exposed to streptavidin peroxidase (Cat: 859043, Lot: 903532A, Invitrogen, USA) for 10 minutes. Then AEC (3-Amino-9-EthylCarbazole, Lot: 911667A, Invitrogen, USA) was used as chromogen. Afterwards, the slides were counterstained with Mayer's haematoxylin. Slides were examined with Photo-light microscope (DCM4000 Image Analyze System, DFC280 Plus Camera and QWin Programme, Leica, Weetlar, Germany).

\section{Results}

The walls of the venae centralis, the surrounding connective tissue and the hepatocytes in radial arrangement were observed to be normal in the control group. The structures of nuclei and nucleoli of hepatocytes and the sinusoids were also normal. While the immunohistochemical staining revealed a weak or intermediate iNOS expression in hepatocytes around venae centralis, the

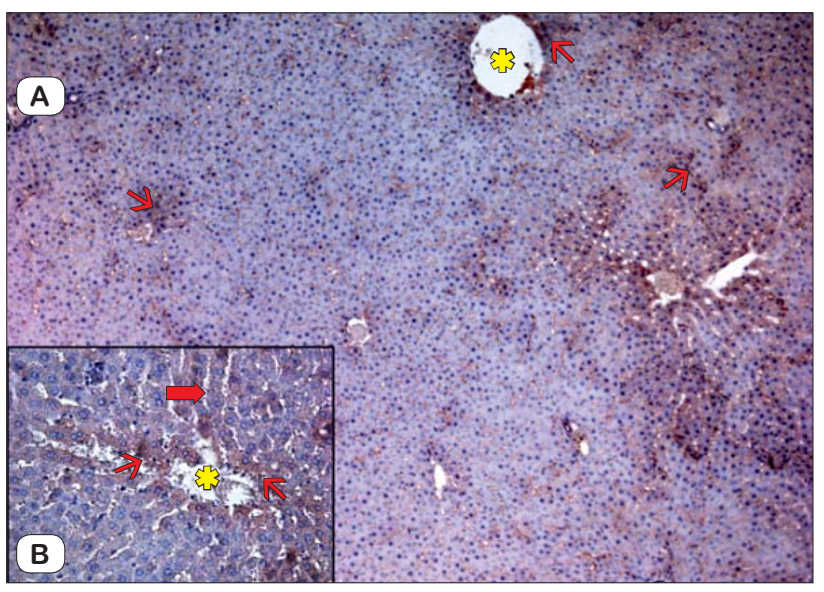

Fig. 2 A,B. INOS expression $(\rightarrow)$ in hepatic tissue in the control group *: Vena centralis, $\rightarrow$ : Hepatic chords (Immunoperoxidase-Hematoxylline AX100, BX400). 


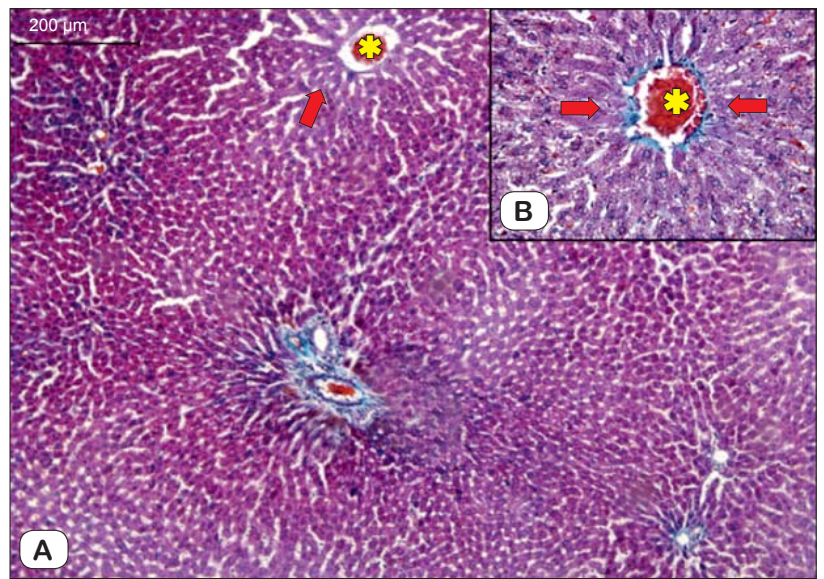

Fig. 3 A,B. Hepatic tissue in Group IR * : Vena centralis, - : Hepatic chords (Masson-Trichrome AX100,BX400).

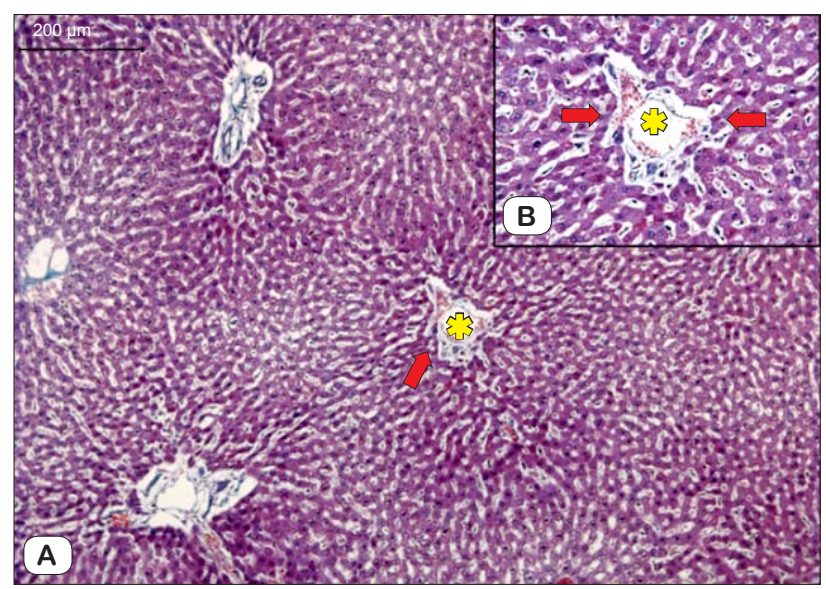

Fig. 5 A,B: Hepatic tissue in Group D * : Vena centralis, - : Hepatic chords (Masson-Trichrome AX100,BX400).

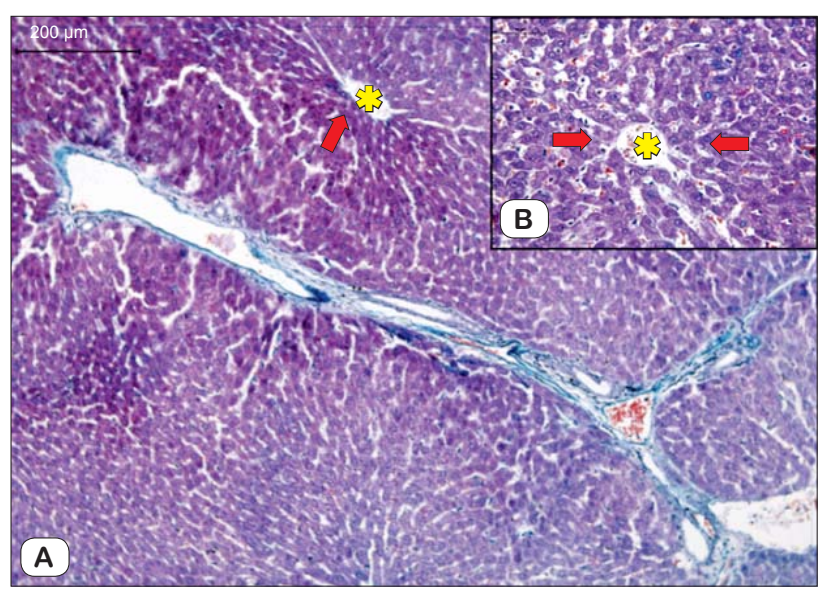

Fig. 7 A,B. Hepatic tissue in Group IR-D *: Vena centralis, - : Hepatic chords (Masson-Trichrome AX100,BX400).

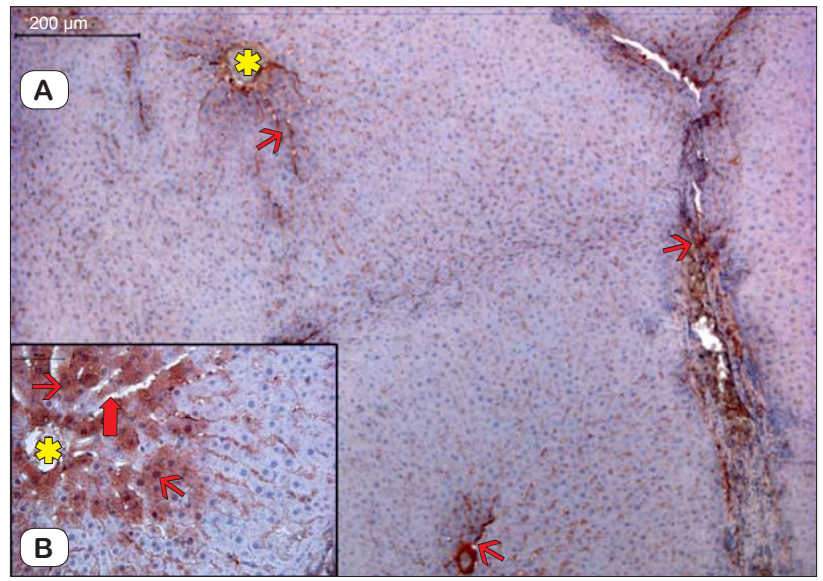

Fig. 4 A,B. INOS expression $(\rightarrow)$ in hepatic tissue in Group IR * : Vena centralis, $\rightarrow$ : Hepatic chords ((Immunoperoxidase-Hematoxylline AX100, BX400).

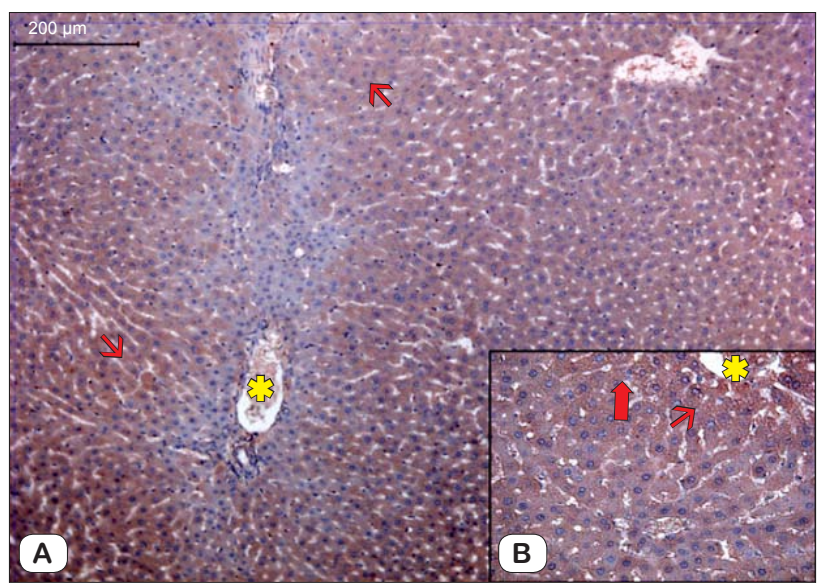

Fig. 6 A,B. INOS expression $(\rightarrow)$ in hepatic tissue in Group D. *: Vena centralis, $\rightarrow$ : Hepatic chords ((Immunoperoxidase-Hematoxylline AX100, BX400).

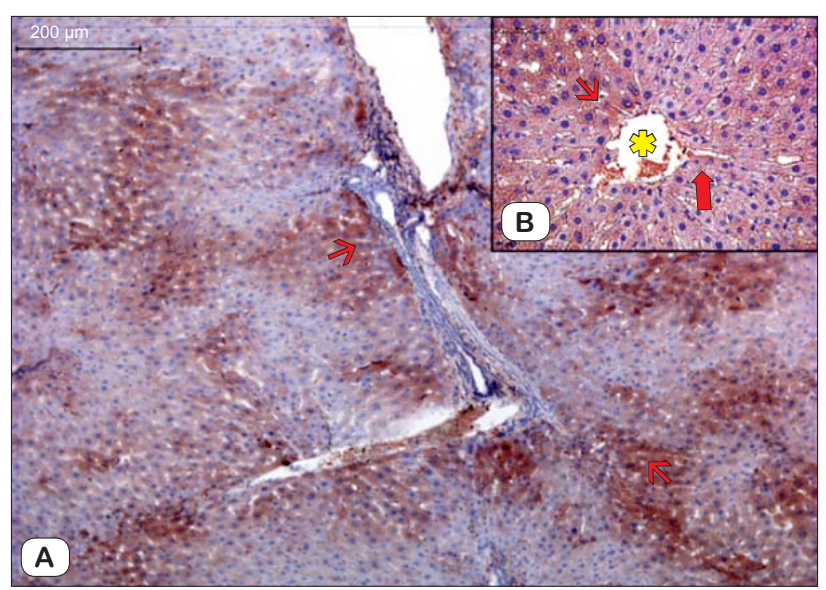

Fig. 8 A,B. INOS expression $(\rightarrow)$ in hepatic tissue in Group IR-D * : Vena centralis, $\rightarrow$ : Hepatic chords ((Immunoperoxidase-Hematoxylline AX100, BX400). 
immunoreaction was negative in other hepatocytes (Figs $1 \mathrm{~A}, \mathrm{~B}$ and $2 \mathrm{~A}, \mathrm{~B})$.

In Group IR, an increase in the connective tissue around venae centralis and degeneration in the endothelial cells were noted. An impairment in the radial arrangement of hepatocytes and cytoplasmic fragmentation were observed. The structures of the nuclei and the nucleoli of the hepatocytes were also found to be impaired. There was a significant decrease in sinusoids, and a structural degeneration was noted. iNOS expression was stronger particularly in vessel walls and perisinusoidal space, and the hepatocytes around venae centralis was found to be increased when compared with the control group (Figs 3 A,B and 4 A,B).

In Group D, a significant perivascular oedema around all venae centralis and sinusoidal dilation were noted. A diffuse degeneration and an impairment in the radial arrangement of the hepatocytes were observed. In the assessment of iNOS expression, an atypically strong immunoreaction decreasing peripherally in the cytoplasm of hepatocytes was detected in this group (Figs 5 A,B and 6 A,B).

A decreased perivascular oedema was observed in Group IR-D compared to Group IR. It was also noted that the impairment in the radial arrangement of the hepatocytes significantly recovered in Group IR-D. The immunoreactivity was found to be significantly decreased in the assessment of iNOS expression in the same group (Figs 7 A,B and 8 A,B).

\section{Discussion}

Ischemia is mainly a local event, but after revascularization, the mediators from the ischemic tissue enter the systemic circulation and affect other organ systems. It has been concluded that the systemic effects of IR injury are caused by activated neutrophils, complement system, and proinflammatory and vasoactive mediators such as eicosanoids, nitric oxide, cytokines, and oxygen free radicals $(19,20)$. The remote effects of IR are most frequently observed in the pulmonary, renal, hepatic and cardiovascular systems, and can result in the development of the systemic inflammatory response and multiple organ dysfunction syndromes, both of which account for $30-40 \%$ of the mortality in intensive care units $(21,22)$.

In the pathophysiology of IR-induced end-organ injury, all authors agree with the significance of oxidant/antioxidant status. For instance, Parlakpinar et al (23) reported that IR plays a causal role in kidney injury, and aminoguanidine (a specific iNOS inhibitor) exerts renal-protective effects, probably by inhibiting NO production and antioxidant activities. Colak et al suggested that melatonin may have protective effects against hepatic injury induced by myocardial IR (4).

We suggest that the administration of dexmedetomidine may have positive effects on liver injury induced by myocardial IR. The effect of dexmedetomidine in IR models depends on several factors such as the route of administration (intravenous (24), intraperitoneal $(15,17)$, dosage, type of ischemic tissue or organ, duration and temperature of ischemia, timing of administration (before $(15,17,25)$ or after $(16)$ ischemia), and the species itself.
Therefore, the effects of anesthetic or sedative agents possibly already being used in anesthesia become an important issue for reducing the IR injury (15, 26-29)

Kocoglu et al suggested that dexmedetomidine was associated with reduced infarct size in IR injury in regional ischemia, but had no effect on the incidence of arrhythmias in a rat model (15).

We determined that administration of dexmedetomidine enhanced erythrocyte deformability during hepatic IR injury in a previous experimental study (26).

Tufek et al (27) demonstrated that dexmedetomidine markedly reduced the oxidative stress in serum, liver, and remote organs induced by hepatic IR injury, and ameliorated the histopathological damage in liver.

Inducing testicular injury by torsion, Shiraishi et al (30) found that NO production by iNOS contributed to necrotic or apoptotic germ cell death in rats. The overexpression of iNOS that produces a toxic level of NO has also been previously reported in apoptotic germ cells of mice testis (31). Similarly, we found significant elevations in iNOS levels in liver tissues after myocardial IR injury. These pathological findings were well correlated with previously reported studies.

Hanci et al (28) suggested that the other mechanisms may explain the effect of dexmedetomidine on endothelial nitric oxide synthase (eNOS) and iNOS levels. In this study, eNOS and iNOS levels were found to be significantly lower in dexmedetomidine groups compared with torsion/ detorsion (T/DT) groups.

Previous studies reported that another anesthetic agent, propofol, attenuates germ cell-specific apoptosis and decreases NO biosynthesis through downregulation of iNOS expression in testicular T/DT (32) .

Tufek et al (27) investigated the effects of dexmedetomidine on kidney and lung injury induced by hepatic IR and Gu et al (33) carried out an experimental study about the effects of dexmedetomidine on lung injury induced by renal IR previously.

The upregulation of iNOS in Group IR means that IR process stimulates DNA damage. However, in Group IR-D, it was shown that iNOS was significantly downregulated. This finding shows that dexmedetomidine has protective effects on IR-related cell damage. Furthermore, the decrease in iNOS expression in Group IR-D also shows the beneficial effect of dexmedetomidine on cellular stress.

In conclusion, the results of this study clearly demonstrate that iNOS is significantly upregulated in experimental liver injury induced by myocardial IR in rats. However, it was observed that dexmedetomidine may have protective effects on these alterations in liver injury when given before induction of ischemia. Other aspects of these findings including clinical significance and practical applications merit further experimental and clinical investigation.

\section{References}

1. Olszanski DA, Ning XH, Childs KF et al. Precursor trapping: A 'Neonatal' mechanism of myocardial protection. J Surg Research 1993; 54: 539.

2. Kleinschmidt S, Baver M, Grundmann U et al. Effect of gammahydroxybutyric acid and pentoxifylline on kidney function parameters in coronary surgery interventions. Anaesthesiol Reanim 1997; 22: 102. 
3. Diaz GC, Moltra V, Sladen RN. Hepatic and renal protection during cardiac surgery. Anesthesiol Clin 2008; 26 (3): 565.

4. Colak C, Parlakpinar H, Ozer MK et al. Investigating the protective effect of melatonin on liver injury related to myocardial ischemia-reperfusion. Med Sci Monit 2007; 13(11): BR251

5. Jaeschke H. Mechanisms of Liver Injury. II. Mechanisms of neutrophilinduced liver cell injury during hepatic ischemia-reperfusion and other acute infl ammatory conditions. Am J Physiol Gastrointest Liver Physiol 2006; 290 (6): G1083.

6. Giakoustidis D, Papageorgiou G, Iliadis S et al. The Protective Effect of alpha-Tocopherol and $\mathrm{GdCl}(3)$ Against Hepatic Ischemia/Reperfusion Injury. Surg Today 2006; 36 (5): 450.

7. Decker K. Biologically active products of stimulated liver macrophages (Kupffer cells). Eur J Biochem 1990; 192: 245.

8. Hou G. Quin P, Ren G. Effect of anthocyanin-rich extract from black rice (Oryza sativa L. Japonica) on chronically alcohol-induced liver damage in rats. J Agric Food Chem 2010; 58; 3191.

9. Lapeyre-Mestre M, de Castro AM, Bareille MP et al. Non-steroidal antiinflammatory drug-related hepatic damage in France and Spain: analysis from national spontaneous reporting systems. Fundam Clin Pharmacol 2006; 20 (4): 391.

10. Jaeschke H, Williams CD, Ramachandran A et al. Acetaminophen hepatotoxicity and repair: the role of sterile inflammation and innate immunity. Liver Int 2012; 32 (1): 8.

11. Morariu AM, Loef BG, Aarts LP et al. Dexamethasone: benefi t and prejudicefor patients undergoing on-pump coronary artery bypass grafting:a study on myocardial, pulmonary, renal, intestinal, and hepaticinjury. Chest 2005;128 (4): 2677.

12. McCutcheon CA, Orme RM, Scott DA et al. A comparison of dexmedetomidine versus conventional therapy for sedation and hemodynamic controlduring carotid endarterectomy performed under regional anesthesia. Anesth Analg 2006; 102: 668-675.

13. Ramsay MA, Luterman DL. Dexmedetomidine as a total intravenous anesthetic agent. Anesthesiology 2004; 101: 787.

14. Maier CM, Sun GH, Kunis DM et al. Neuroprotection by the Nmethyl-D-aspartate receptor antagonist CGP 40116: In vivo and in vitro studies. J Neurochem 1995; 65: 652

15. Kocoglu H, Karaaslan K, Gonca $E$ et al. Preconditioning effects of dexmedetomidine on myocardial ischemia/reperfusion injury in rats. Curr Ther Res Clin Exp 2008; 69: 150.

16. Kocoglu H, Ozturk H, Ozturk $H$ et al. Effect of dexmedetomidine on ischemia-reperfusion injury in rat kidney: a histopathologic study. Ren Fail 2009; 31 (1): 70.

17. Hoffman WE, Kochs E, Werner C et al. Dexmedetomidine improves neurologic outcome from incomplete ischemia in the rat. Reversal by the alpha 2- adrenergic antagonist atipamezole. Anesthesiology 1991; 75: 328.

18. Snapir A, Posti J, Kentala E et al. Effects of low and high plasma concentrations of dexmedetomidine on myocardial perfusion and cardiac function in healthy male subjects. Anesthesiology 2006; 105: 902.
19. Bonanno FG. Clinical pathology of the shock syndromes. J Emerg Trauma Shock 2011; 4 (2): 233.

20. LaNoue JL, Turnage RH, Kadesky KM et al. The effect of intestinal reperfusion on renal function and perfusion. J Surg Res 1996; 64: 19.

21. Sucu N, Unlu A, Tamer $\mathbf{L}$ et al. Effects of trimetazidine on tissue damage in kidneyafter hindlimb ischemia-reperfusion. Pharmacol Res 2002; 46: 345 .

22. Esme H, Fidan H, Koken T et al. Effect of lung ischemia-reperfusion on oxidative stress parameters of remote tissues. Eur J Cardiothorac Surg 2006; 29 (3): 294

23. Parlakpinar H, Ozer MK, Cicek E et al. Renal damage in rats inducedby myocardial ischemia/reperfusion: Role of nitric oxide. Int J Urol 2006; 13 (10): 1327.

24. Kakinohana M, Oshiro M, Saikawa S et al. Intravenous infusion of dexmedetomidine can prevent the degeneration of spinal ventral neurons induced by intrathecal morphine after a noninjurious interval of spinal cord ischemia in rats. Anesth Analg 2007; 105 (4): 1086

25. Engelhard K, Werner $\mathbf{C}$, Eberspächer $\mathbf{E}$ et al. The effect of the alpha 2-agonist dexmedetomidine and the N-methyl-D-aspartate antagonist $\mathrm{S}(+)$-ketamine on the expression of apoptosis-regulating proteins after incomplete cerebral ischemia and reperfusion in rats. Anesth Analg 2003; 96 (2): 524.

26. Arslan M, Comu FM, Kucuk A et al. Dexmedetomidine protects against lipid peroxidation and erythrocyte deformability alterations in experimental hepatic ischemia reperfusion injury. Libyan J Med. 2012;7. doi: 10.3402/ljm.v7i0.18185. Epub 2012 May 24.

27. Tüfek A, Tokgöz $\mathbf{O}$, Aliosmanoglu I et al. The protective effects of dexmedetomidine on the liver and remote organs against hepatic ischemia reperfusion injury in rats. Int J Surg 2012 Dec 20. pii: S17439191(12)00850-3. doi: 10.1016/j.ijsu.2012.12.003. (Epub ahead of print).

28. Hanci V, Erol B, Bektas S et al. Effect of dexmedetomidine on testicular torsion/detorsion damage in rats. Urol Int 2010; 84 (1): 105.

29. Inci FDI, Eti Z. The effects of dexmedetomidine infusion on the formation of reactive oxygen species during mesenteric ischemia-reperfusion injury in rats. Marmara Med J 2007; 20 (3): 154.

30. Shiraishi K, Naito K, Yoshida K. Nitric oxide promotes germ cell necrosis in the delayed phase after experimental testicular torsion of rat. Biol Reprod 2001; 65: 514.

31. Lue Y, Sinha Hikim AP, Wang C et al. Functional role of inducible nitric oxide synthase in the induction of male germ cell apoptosis, regulation of sperm number, and determination of testes size: evidence from null mutant mice. Endocrinology 2003; 144: 3092.

32. Yagmurdur H, Ayyildiz A, Karaguzel E et al. Propofol reduces nitric oxide-induced apoptosis in testicular ischemia-reperfusion injury by downregulating the expression of inducible nitric oxide synthase. Acta Anaesthesiol Scand 2008; 52: 350.

33. Gu J, Chen J, Xia P et al. Dexmedetomidine attenuates remote lung injury induced by renal ischemia-reperfusion in mice. Acta Anaesthesiol Scand 2011; 55 (10): 1272. 